\title{
VIRTUAL BANDWITH SAR (VB-SAR) FOR CENTIMETER-SCALE VERTICAL PROFILING THROUGH A SOIL AT C-BAND FROM SPACE
}

\author{
Keith Morrison ${ }^{1}$, Alexander Edwards-Smith ${ }^{1}$, Simon Zwieback ${ }^{2}$, and Irena Hajnsek ${ }^{2,3}$ \\ 1: Centre for Electronic Warfare, Cranfield University, Swindon, UK \\ 2: Institute of Environmental Engineering, ETH Zurich, Zurich, Switzerland \\ 3: Microwaves and Radar Institute, German Aerospace Center (DLR-HR), Oberpfaffenhofen, Germany
}

\begin{abstract}
The first experimental demonstration of the Virtual Bandwidth SAR (VB-SAR) scheme is provided. VB-SAR is a new technique that promises subsurface imaging of soils at ultra-high, centimeter-scale resolution at large stand-off distances applicable to aircraft and spacecraft. This paper reports on how a stack of C-band images were used to retrieve high resolution vertical profiles of the backscattering through a soil in the laboratory. The VB-SAR scheme captures the phase behavior of a soil across a stack of DInSAR images as the soil dries. The real frequency of the interrogating radar behaves as a higher, virtual frequency within the soil by virtue of its higher-than-air dielectric. As the dielectric changes with time, the DInSAR stack captures a virtual bandwidth. Using this scheme, it was possible to produce a vertical slice of the backscatter through a soil at $10 \mathrm{~cm}$ resolution, much improved on the formal $1 \mathrm{~m}$ resolution offered by the real $150 \mathrm{MHz}$ bandwidth.
\end{abstract}

Index Terms - VB-SAR, virtual bandwidth, soil moisture, soil dielectric, DInSAR

\section{INTRODUCTION}

Virtual Bandwidth SAR (VB-SAR) is a novel technique that promises to open up a new remote sensing capability in subsurface imaging at large stand-off distances applicable to aircraft and spacecraft [1]. Ground-based and near-ground systems currently provide the only practical method for high-resolution, subsurface imaging. These systems rely on close proximity measurement in order to spatially isolate vertically the surface and subsurface backscatter returns [2]. The VB-SAR scheme exploits the change in soil dielectric properties which occur when a soil's moisture changes. A real radar wave entering a soil is compressed and behaves like a wave at a higher frequency. As it dries, the real radar frequency is sequentially transported across a virtual bandwidth of virtual frequencies. Even a moderate soil moisture change (SMC) can produce large virtual bandwidths; an SMC change of $10 \%$ can provide $3 \mathrm{~cm}$ vertical resolution at $\mathrm{C}$-band, and double that at $\mathrm{X}$-band. In addition, the VB-SAR scheme provides an unambiguous discrimination between surface and subsurface returns. Only objects within the soil will respond in sympathy to the changes in SMC. Above ground will not experience a virtual bandwidth and so will appear at zero depth in a reconstruction. Implemented from an aircraft or spacecraft platform, it would require collection of a stack of conventional DInSAR images over an SMC change. The differential phase history at each image pixel would be processed to produce a VB-SAR depth profile at that pixel. Repeating this across the image would provide a 3D map of the soil backscatter. This paper presents the results of a laboratory experiment which provides the first experimental demonstration of VB-SAR capability, and validation of the scheme

\section{MEASUREMENTS}

The measurements were carried out at Cranfield University's Ground-Based SAR (GB-SAR) Microwave Measurement Facility. In this system a $4 \mathrm{~m}$ linear scanner sits centrally along the length of a $4 \mathrm{~m} \times 1 \mathrm{~m} \times 0.9 \mathrm{~m}$ (h) soil trough. Imagery is obtained by driving the antenna cluster under computer control along the length of the scanner. Figure 1 shows a view of the scanner above the soil trough. The trough was filled with fine kiln-dried sand, aside from a centrally-placed $1 \mathrm{~m} \times 1 \mathrm{~m}$ soil-gravel region which extended $26 \mathrm{~cm}$ downwards from the surface. It consisted of a mixture of $10 \%$ gravel and $90 \%$ sand by volume. The randomlyshaped gravel pieces were nominally around $1 \mathrm{~cm}$ in size. At the base of the mixture a trihedral was placed and angled to give a maximum return for the imaging geometry. The surface of the sand was level and smooth. Several reference trihedrals were placed on the surface of the sand in order to allow monitoring and correction of any unwanted systemrelated phase and amplitude variations.

The antenna platform of the trough scanner was positioned to give a maximum illumination for an incidence angle of $20^{\circ}$. For each scan, the platform moved along a $300 \mathrm{~cm}$ long aperture, taking a sample every $2 \mathrm{~cm}$ for a total of 151 samples per scan. C-band measurements at VV polarization were made using a bandwidth of $2 \mathrm{GHz}$ over the 


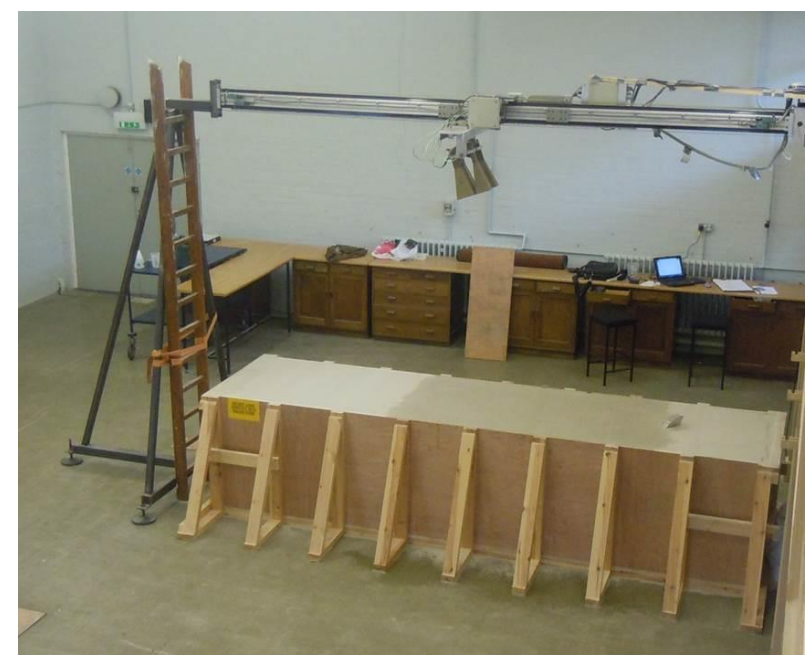

Figure 1: View of the laboratory, showing the linear scanner mounted centrally along the length of the sand trough.

interval 4-6GHz. Subsequently, water was sequentially added only to the $1 \mathrm{~m} \times 1 \mathrm{~m}$ surface area corresponding to the sand/gravel mixture in precisely controlled amounts. After each addition the scene was re-imaged. These wetting scans were used to characterize the phase response of the buried trihedral to a change in SMC. In total, $28000 \mathrm{ml}$ of water was added. Assuming a homogeneous distribution of moisture within the gravel-sand volume, and neglecting exfiltration of water away from this volume, this corresponded to a maximum SMC within the wetted volume of $10.6 \%$. After the water addition was complete, the entire scene was left to dry whilst being reimaged approximately every 80 minutes over a 5-week period. The SMC at the end of the 5-week drying period was calculated to be $4.3 \%$.

The DInSAR stack was gathered using tomographic

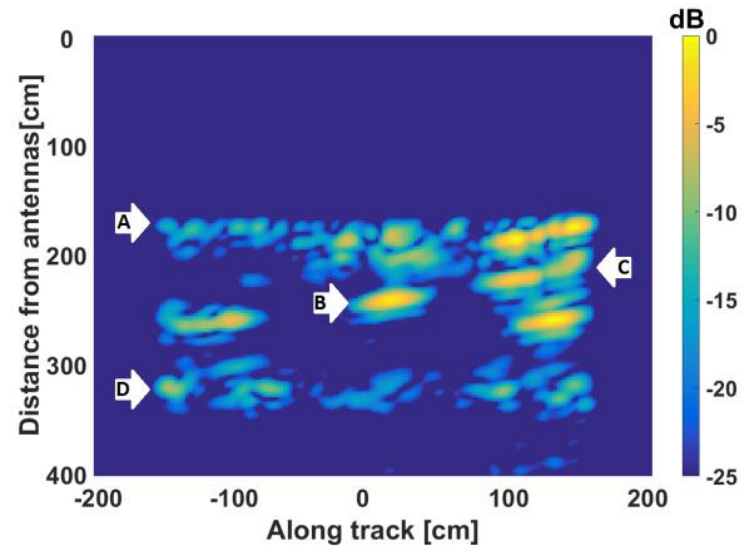

Figure 2: Example high resolution TP image using the entire real bandwidth. $A$ indicates the soil surface, with the strong returns at right being generated by surface reference trihedrals, $B$ shows the buried trihedral. $C$ and $D$ are features related to other objects present in the soil not used in this study. profiling (TP) [3]. This process produces focused complex radar images by a means of synthetic beam forming across a synthetic aperture. It provides vertical looks through a target volume along a transect directly below the scanner. Constant re-positioning of the antenna ensures the imaging geometry is identical at each pixel. This image product enables the complex development of the soil and its internal features to be studied and extracted over the TP image set.

\section{IMAGERY}

The data was processed at two slant range resolutions; the maximum of $7.5 \mathrm{~cm}$ allowed by the $2 \mathrm{GHz}$ bandwidth collected and an artificially lowered slant range resolution of $1 \mathrm{~m}$ using the first $150 \mathrm{MHz}$ of the bandwidth. In both cases the cross-range (x-axis) resolution was the same. A sample high resolution image is shown in Figure 2, and the same image processed at low range resolution is shown in Figure 3. In Figure 2, the soil surface appears at $165 \mathrm{~cm}$ below the antennas, and the buried trihedral is the strong isolated below-ground response close to $0 \mathrm{~m}$ cross-range. The two returns at the top right of the soil volume are two of the surface reference trihedrals. With TP imaging we have the luxury of being able to isolate and monitor the phase response of the pixel containing the response of the buried trihedral. By monitoring this phase change at the trihedral during the wetting process - and knowing the SMC change a SMC-phase relationship was derived. This was observed to be linear, in agreement with [1]. This then informed on changes in SMC during the drying scans.

For the demonstration of the VB-SAR scheme, a set of $1 \mathrm{~m}$ low slant range resolution images were generated across the DInSAR stack. By producing TP images with dramatically reduced range resolution it was possible to emulate side looking SAR data as might be acquired from an air or spaceborne SAR system where one pixel represents the entire surface and subsurface column collapsed into a ground pixel. For the low resolution case, Figure 3 shows

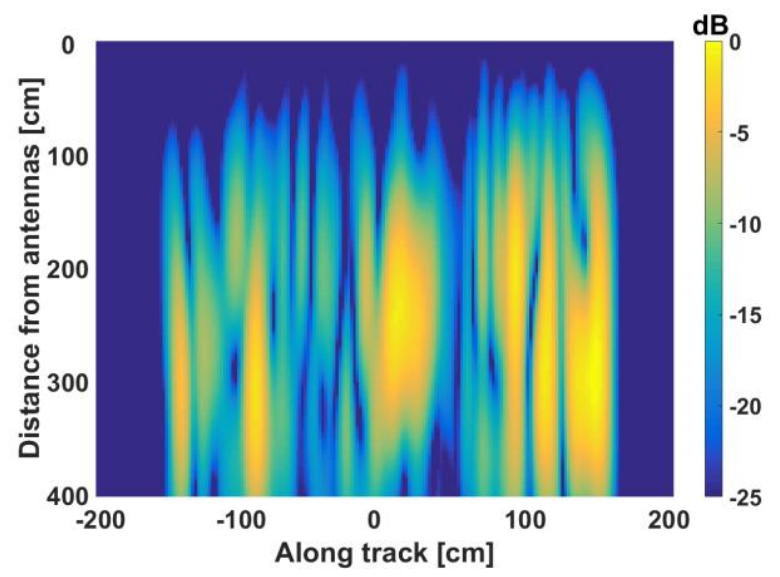

Figure 3: Example of a low range resolution TP image created using a $150 \mathrm{MHz}$ fraction of the real bandwidth. 
that the surface and volume returns are no longer separable.

\section{VB-SAR ANALYSIS}

VB-SAR, as outlined in [1], promises to allow subsurface imaging at enhanced range resolutions by exploiting the variability of the dielectric properties of a soil with SMC (as described by [4]). The core principle behind VB-SAR is that a radar wave of a given real frequency, $f_{r}$, entering a soil volume behaves as if it were a wave of a higher "virtual" frequency, $f_{v}$, due to the soil's refractive index being higher than 1. Over a series of radar images the refractive index of the soil will change due to SMC variation. This implies that over the series of images the virtual frequency also varies which leads to a virtual bandwidth, $B_{v}$, being synthesized. The virtual bandwidth generated can be calculated by;

$$
B_{v}=f_{r} \Delta \sqrt{\varepsilon}
$$

where $\Delta \sqrt{\varepsilon}$ is the variation is the refractive index during the measurements, and $\varepsilon$ is the relative dielectric constant. The phase history extracted from the buried trihedral using the full resolution imagery during the drying period is shown in Figure 4. It is clear that the phase history is non-linear with time. This is presumed to be due to uneven drying rates; in the early drying period it is reasonable to assume that the rate of SMC was higher due to higher evaporation. However, it is possible to resample this history to a linear SMC change using the SMC-phase relationship derived above from the wetting measurements. After linearization in $\mathrm{SMC}$, the resampled complex (phase and amplitude) low resolution data was passed to an FFT. The phase history stack at each pixel produces a single depth profile through the soil at that point. This is shown in Figure 5 at the pixel corresponding to the trihedral position. In contrast, to the low resolution result in Figure 3, the surface and buried trihedral appear resolved. Both appear at the correct depths of $0 \mathrm{~cm}$ and $26 \mathrm{~cm}$, respectively. By repeating this along the

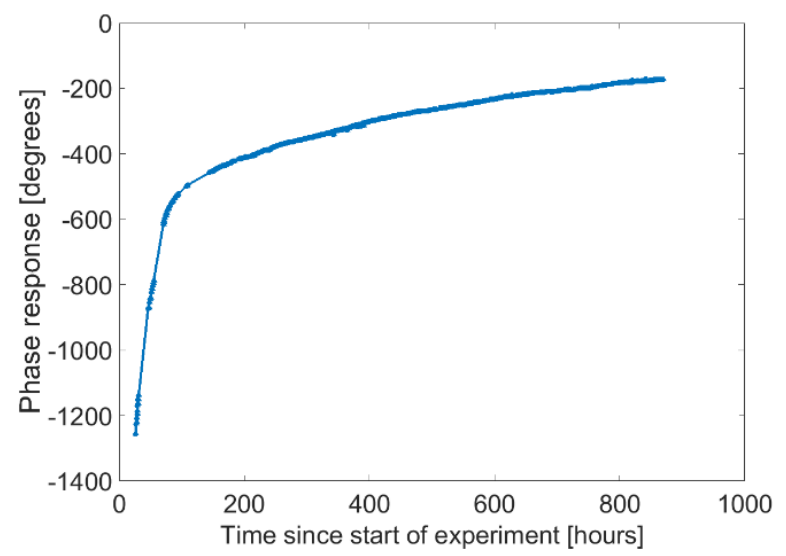

Figure 4: The phase history of buried trihedral over the drying phase.

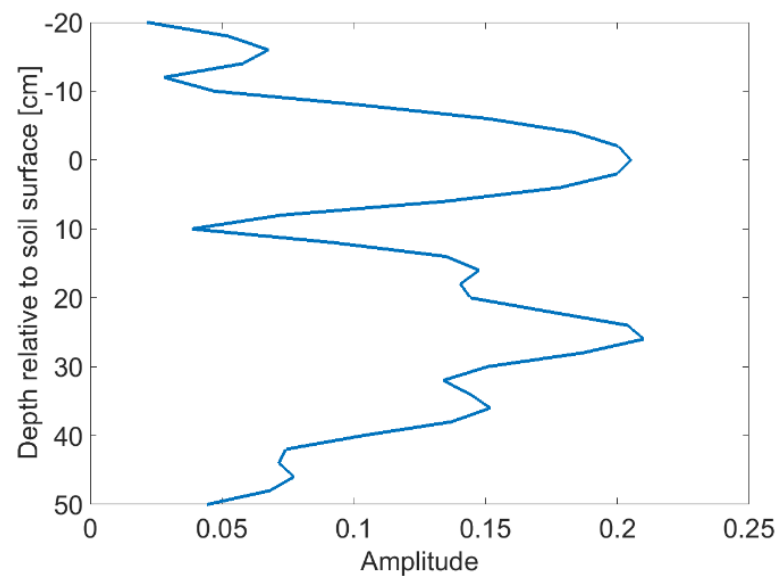

Figure 5: The depth profile produced from an FFT of the phase history at the pixel corresponding to the position of the buried trihedral (ie using the SMC-linearized phase shown in Figure 4).

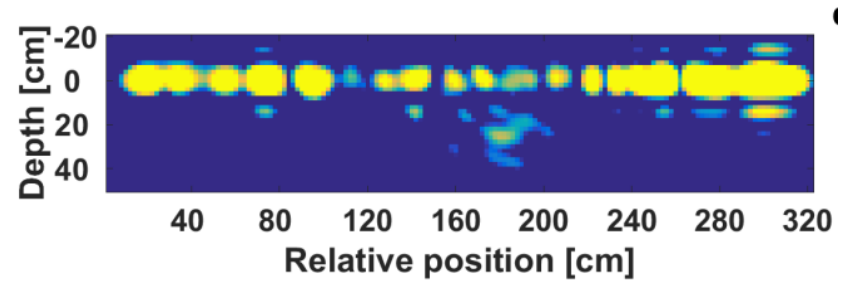

Figure 6: The final VB-SAR image along the $3 \mathrm{~m}$ transect derived from the low resolution DInSAR stack.

transect, the complete VB-SAR image is produced in Figure 6 , showing the vertical profile through the soil along the transect. The surface appears at $0 \mathrm{~cm}$ along the whole $3 \mathrm{~m}$ transect, although only the central $1 \mathrm{~m}$ was wet during the measurements. Nevertheless, both regions see a surface return at $0 \mathrm{~cm}$. Note that in the dry areas at the sides of the image scene all targets including those buried within the soil appear at zero depth. This is evidence that the VB-SAR process is working as expected as the theory states that in areas that experience no SMC variation no virtual bandwidth is synthesised and so a completely flat phase history is presented to the VB-SAR processor. This gives a peak at zero depth. All returns from all depths in the dry areas are effectively summed together and placed at zero depth, hence the strong surface return seen in Figure 6. The only significant subsurface feature present is that associated with the buried trihedral, which appears at the correct depth.

\section{SUMMARY}

This study has described a laboratory demonstration of VBSAR imaging. An image dataset which captured a controlled increase and then decrease in SMC was collected in the laboratory under carefully controlled conditions.

From this, the basic principles of VB-SAR processing have been validated. Namely, a virtual bandwidth can be created and used to significantly enhance the subsurface range resolution compared to that produced using the formal range resolution generated by the radar's real bandwidth. In 
addition, the direct and unambiguous separation of surface and subsurface features was demonstrated.

There are undoubtedly additional considerations and complications to the real-world application of the VB-SAR scheme. An absence of knowledge of SMC values can be anticipated for wide-area imaging collected from air or spaceborne platforms. Non-linear sampling will compromise the performance of the scheme, and a knowledge of absolute SMC is necessary to provide an accurate depth scale. However, many conventional amplitude-based SMC retrieval schemes have been proposed [5] which can help fill the information gap.

\section{REFERENCES}

[1] Morrison, K. and Bennett, J. Virtual bandwidth SAR (VB-SAR) for centimetric-scale sub-surface imaging from space, International Journal of Remote Sensing, vol. 36, no. 7, pp. 1789-1808, 2015.

[2] D.J. Daniels. Ground Penetrating Radar, Encyclopedia of RF and Microwave Engineering, John Wiley \& Sons, 2005.

[3] Morrison, K. and Bennett, J. Tomographic profiling - A technique for multi-incidence-angle retrieval of the vertical SAR backscattering profiles of biogeophysical targets, IEEE Transactions on Geoscience and Remote Sensing, vol. 52, no. 2, pp. 1250-1255, 2014.

[4] Hallikainen, M.T. and Ulabz, F.T. and Dobson, M.C. and El-Rayes, M.A. and Wu, L.-K. Microwave Dielectric Behavior of Wet Soil-Part I: Empirical Models and Experimental Observations IEEE Transactions on Geoscience and Remote Sensing, vol. GE-23, no. 1, pp. 2534, 1985.

[5] Dubois, P.C., van Zyl, J., Engman, T. Measuring soil moisture with imaging radars, IEEE Trans. Geosci. Remote Sens., IEEE, 1995. 